\title{
¿Cumple el gravamen a los movimientos financieros en Colombia con los principios de regresividad y equidad?
}

\author{
Luisa Fernanda Briceño-Forero ${ }^{1}$ \\ Escuela Colombiana de Ingeniería Julio Garavito \\ luisa.briceno@mail.escuelaing.edu.co \\ Andrés Felipe Cañón-Moreno ${ }^{2}$ \\ Escuela Colombiana de Ingeniería Julio Garavito \\ andres.canon@mail.escuelaing.edu.co
}

\section{DOI: https://doi.org/10.21158/23227230.v10.n0.2020.2946}

Cómo citar este artículo: Briceño-Forero, L. F.; Cañón-Moreno, A. F. (2020). ¿Cumple el gravamen a los movimientos financieros en Colombia con los principios de regresividad y equidad? Revista Ploutos, 10, (Páginas). DOI: https://doi.org/10.21158/23227230.v10.n0.2020.2946

Fecha de recepción: 02 de octubre de 2020

Fecha de aprobación: 09 de abril de 2021

\section{Resumen}

Este trabajo analiza la evolución de los indicadores de productividad - efectividad de recaudo-y progresividad - aporte a la equidad - del gravamen a los movimientos financieros (GMF), con el fin de analizar si este impuesto ha cumplido con los principios tributarios de regresividad y equidad. El objetivo del estudio es realizar un análisis detallado de la estructura del GMF para el periodo 1999-2018 a través de la construcción del indicador de progresividad/regresividad y del indicador de productividad, para así visualizar el comportamiento del recaudo respecto al producto interno bruto. De igual forma, se busca ejemplificar través de un impuesto indirecto la senda que ha perseguido la política tributaria en Colombia entre 1999 y el 2018, la cual, de acuerdo con la Constitución Política de 1991, debe procurar aportar a la equidad. La teoría sugiere que los impuestos indirectos tienen ventajas en productividad del recaudo, pero efectos negativos en términos de progresividad. No obstante, los indicadores examinados para el impuesto del GMF sugieren una mejora en progresividad mientras su productividad se disminuye, lo cual demuestra que es un impuesto que sacrifica productividad para ganar progresividad.

Palabras clave: gravamen a los movimientos financieros (GMF); indicadores de productividad; indicadores de progresividad; política tributaria; principio de regresividad; principio de equidad; efectividad de recaudo.

\footnotetext{
${ }^{1}$ Economista con énfasis en finanzas - Escuela Colombiana de Ingeniería Julio Garavito. Integrante del Semillero de investigación finanzas y economía. ORCID: https://orcid.org/0000-0001-9254-8193

2 Economista con énfasis en finanzas - Escuela Colombiana de Ingeniería Julio Garavito. Integrante del Semillero de investigación finanzas y economía. ORCID: https://orcid.org/0000-0002-1481-8378 


\title{
Does the tax on financial movements in Colombia
} comply with the principles of regressivity and equity?

\begin{abstract}
This paper analyzes the evolution of the productivity indicators - collection effectiveness - and progressivity contribution to equity - in the tax on financial movements (GMF by its abbreviation in Spanish), in order to investigate whether this tax has complied with the tax principles of regressivity and equity. The aim of the study is to perform a detailed analysis of the GMF structure for the period 1999-2018, by the construction of the progressivity/regressivity indicator and the productivity indicator, in order to visualize collection behavior with respect to gross domestic product. Also, and by means of an indirect tax, we seek to exemplify the path that tax policy in Colombia pursued between 1999 and 2018, which, according to the Political Constitution of 1991, should have sought to contribute to equity. The theory suggests that indirect taxes have advantages in terms of collection productivity, but negative effects in terms of progressivity. However, the indicators examined for the GMF tax suggest an improvement in progressivity while productivity decreases, showing that this is a tax that sacrifices productivity to gain progressivity.
\end{abstract}

Keywords: tax on financial movements (GMF by its abbreviation in Spanish); productivity indicators; progressivity indicators; tax policy; principle of regressivity; principle of equity; collection effectiveness.

O imposto sobre movimentos financeiros na Colômbia

obedece aos princípios de regressividade e equidade?

\section{Resumo}

Este trabalho analisa a evolução dos indicadores de produtividade - eficácia da arrecadação - e progressividade contribuição para a equidade - do imposto sobre movimentos financeiros (do espanhol GMF), a fim de analisar se esse imposto obedece aos princípios tributários da regressividade e da equidade. $O$ objetivo do estudo é realizar uma análise detalhada da estrutura do GMF para o período de 1999-2018 por meio da construção do indicador de progressividade/regressividade e do indicador de produtividade, a fim de visualizar o comportamento da arrecadação em relação ao produto interno bruto. Da mesma forma, busca exemplificar, por meio de um imposto indireto, a trajetória que a política tributária percorreu na Colômbia entre 1999 e 2018, que, de acordo com a Constituição Política de 1991, deve buscar contribuir para a equidade. A teoria sugere que os impostos indiretos têm vantagens na produtividade da arrecadação, mas efeitos negativos em termos de progressividade. No entanto, os indicadores examinados para o imposto GMF sugerem uma melhora na progressividade enquanto sua produtividade diminui, o que mostra que se trata de um imposto que sacrifica a produtividade para ganhar progressividade.

Palavras-chave: imposto sobre movimentos financeiros (do espanhol GMF), indicadores de produtividade, indicadores de progressividade, política tributária, princípio de regressividade, princípio da equidade, eficácia de arrecadação. 
La taxe sur les mouvements financiers en Colombie

respecte-t-elle les principes de dégressivité et d'équité?

\begin{abstract}
Résumé
Ce travail analyse l'évolution des indicateurs de productivité - efficacité de collecte - et de progressivité contribution à l'équité - de la taxe sur les mouvements financiers (TMF), pour définir si celle-ci respecte les principes fiscaux de dégressivité et d'équité. L'objectif de cette étude est de réaliser une analyse détaillée de la structure de la TMF sur la période 1999-2018 en construisant un indicateur de progressivité/dégressivité et un indicateur de productivité pour visualiser le comportement de la collecte de cette taxe en rapport au produit intérieur brut. Cet impôt indirect montre le cheminement de la politique fiscale colombienne entre 1999 et 2018, qui doit, selon la Constitution de 1991, contribuer et protéger l'équité nationale. La théorie semble suggérer que les impôts indirects offrent des avantages en termes de productivité des revenus, mais auraient des effets négatifs en termes de progressivité. Les indicateurs analysés pour la TMF suggèrent une amélioration de la progressivité alors que la productivité diminue, révélant qu'il s'agit d'une taxe sacrifiant la productivité pour gagner en progressivité.
\end{abstract}

Mots-clés: taxe sur les mouvements financiers (TMF) ; indicateurs de productivité ; indicateurs de progressivité ; politique fiscale ; principe de dégressivité ; principe d'équité ; efficacité de la collecte.

\title{
1. Introducción
}

En Colombia se ha generado un complejo entorno de discusión alrededor del sostenimiento del gravamen a los movimientos financieros (GMF) y su alcance en términos de eficiencia y equidad, una discusión que, hasta el momento, ha sido enmarcada en un entorno analítico al recurrir siempre a las facultades teóricas que posee el tributo en cuestión, distanciándose de los aspectos empíricos que sugieren contraste con lo mostrado por la teoría. Así mismo, de acuerdo con Steiner y Cañas (2013), fue posible establecer el carácter inequitativo que ha perseguido la política tributaria en Colombia bajo un perfil fiscalista, es decir, la búsqueda amplia de una fuente de financiamiento e ingresos más sólidos, principalmente, del orden tributario, a fin de evitar profundizar la deuda, de manera que se rezaga la idea de buscar equidad.

Al hablar de política tributaria es necesario hacer referencia a la estructura, es decir, entender la conformación del sistema dados los tipos de impuestos, bien sean de orden directo ${ }^{3}$ o bien indirecto4, para la consecución de los objetivos de equidad y eficiencia. Según López, Torres y Molina

\footnotetext{
${ }^{3}$ Aquellos impuestos que recaen directamente sobre la persona - como, por ejemplo, el impuesto a renta, entre otros-.

${ }^{4}$ Aquellos impuestos que no recaen directamente sobre la persona -IVA o GMF, entre otros-.
} 
(2011), a priori se comete el error de enmarcar los impuestos indirectos como regresivos 5 y los directos como progresivos 6 . Por esta razón, es necesario contrastar la suposición teórica con indicadores sujetos a datos reales que conduzcan a la identificación del comportamiento real del tributo.

Siguiendo esta línea de pensamiento, el objetivo de este trabajo es realizar un análisis detallado de la estructura del GMF para el periodo 1999-2018 a través de la construcción del indicador de progresividad/regresividad y del indicador de productividad, con el fin de visualizar el comportamiento del recaudo respecto al producto interno bruto. Esto evidencia el carácter bien sea fiscalista o equitativo que ha seguido la administración tributaria con este gravamen, ya que la estructura teórica del impuesto permitiría inferir que el GMF se ha caracterizado por ser un impuesto eficaz y con alta regresividad. El trabajo de investigación que se realiza presenta un caso particular, ya que en estudios anteriores no se ha realizado el análisis de manera simultánea de los dos tipos de indicadores, el de productividad y el de regresividad del GMF.

Para lograr el objetivo de estudio el artículo se desarrolla en cinco partes adicionales a esta introducción. En la primera sección, se expone el origen del GMF y el contexto que llevó a Colombia a adoptar dicho impuesto en su sistema tributario; la segunda parte le brinda al lector una visión sobre estudios enmarcados en el sistema tributario y la discusión entre impuestos indirectos y directos; la tercera sección esclarece la metodología que se utiliza para realizar los cálculos de los indicadores de productividad y progresividad del GMF, con énfasis en los datos seleccionados para los cálculos que sustentan el trabajo; la cuarta presenta brevemente los resultados obtenidos respecto a la productividad y regresividad del GMF, y, por último, se exponen las conclusiones en torno a la estructura del GMF y su objetivo dentro de la política tributaria de Colombia.

\footnotetext{
${ }^{5}$ Aquellos que cobran una menor tasa impositiva a medida que el ingreso del contribuyente aumenta. ${ }^{6}$ Aquellos que ajustan la carga tributaria de acuerdo con el nivel de ingreso del contribuyente. 


\section{2. ¿Por qué y cómo se incluye el GMF en el sistema tributario colombiano?}

En las décadas de los ochenta y los noventa del siglo pasado, el pensamiento dominante sostuvo la idea de una liberalización de los mercados financieros, sugiriendo una gran cantidad de beneficios como resultado, específicamente en rubros tales como inversión

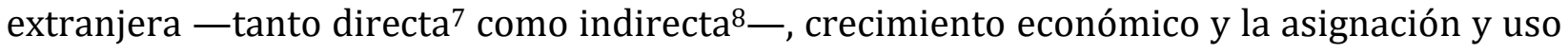
de recursos para iniciar, paulatinamente, un proceso de reducción de la deuda externa; estos tendrían un efecto significativo en las economías mundiales y opacarían cualquier contradicción que pudiera presentarse. Sin embargo, al pasar el tiempo los altos flujos de capital empezaron a tener repercusiones en los mercados internos de los países, lo cual se tradujo en inestabilidad a nivel macroeconómico.

En 1981, el economista keynesiano James Tobin —premio nobel de economía de 1981- se interesó por los efectos derivados de la liberalización financiera. En su análisis del mercado de capitales indicó la importancia de desincentivar los movimientos de capitales a corto plazo. A fin de solucionar este inconveniente, causante de los efectos negativos sobre las economías, propuso implementar un gravamen sobre estos movimientos para regularlos y contrarrestar los efectos negativos de la globalización, lo cual era una buena opción con miras a reducir la volatilidad de los mercados financieros nacionales e internacionales y dar así mayor autonomía a la política económica de los países emergentes.

La propuesta original de James Tobin fue analizada tiempo después por varios estudiosos. Así, por ejemplo, Cornford (1996) indicó que el impuesto planteado por Tobin consistía en instaurar un gravamen uniforme a todas las transacciones financieras que involucrarán un cambio de divisa; al establecer una tarifa del $1 \%$ sobre el valor de la transacción se buscaba desincentivar las transacciones o los movimientos de capital a corto plazo, pues los inversionistas reducirían los movimientos al tener que pagar por cada uno de ellos. Además, menciona que para Tobin esta regulación era menos nociva en comparación con otro tipo de políticas proteccionistas.

\footnotetext{
7 Supone la creación de empresas dentro de un territorio con capitales netamente extranjeros.

${ }^{8}$ Adquisición de acciones, bonos y otras formas de participación que no requieren propiamente la propiedad de extranjeros. Este artículo es un online first (versión definitiva del contenido del artículo, con diagramación provisional y asignación de DOI). Aún no cuenta con páginas definitivas, pero es citable utilizando su código DOI.
} 
Siguiendo la argumentación de Tobin, el GMF figura como una solución de estabilización ante cambios abruptos del mercado de valores y los efectos colaterales que se trasmiten al sistema financiero y a la economía. En general, en la historia de los mercados financieros se han presentado diversos episodios que han desestabilizado el sistema financiero, entre los cuales podemos mencionar la crisis de 1929, el lunes negro de 1987 o, incluso, la burbuja inmobiliaria del 2008. Es posible enunciar más, sin embargo, nos atañe el episodio presentado por la economía colombiana en 1998, en el cual, de acuerdo con ValeroVarela (2007), los efectos de estabilización de banda cambiaron en Brasil y el cese de pagos a obligaciones crediticias con Rusia generaron un proceso de desestabilización vía pérdidas considerables de confianza y flujo de capitales.

Así mismo, Valero-Varela (2007) afirma que esa situación empezó a preocupar a los ahorradores y esta senda deterioró la demanda sobre títulos tanto a mediano como a largo plazo. Incluso, el impulso sobre las tasas de interés que intentó la autoridad monetaria no fue suficiente, de modo que se requirió un ajuste adicional para garantizar la liquidez del sistema financiero. Por esto que surge en Colombia el gravamen a los movimientos financieros, con el fin de garantizar solvencia a los bancos y evitar la caída del sistema financiero colombiano, inicialmente, con una tasa del 2 × $1000-0,2 \%$ -

Con el pasar de los años y gracias a la Ley 633 expedida en el 2000 se establece que el GMF se convierte en un nuevo impuesto de manera permanente en la estructura tributaria de Colombia. Además, por medio de la Ley 863 del 2003 se modifica la tasa de recaudo de dicho gravamen, pasando del 0,2 \% hasta situarse en el 0,4\%. Con esta última acción legal por parte del Gobierno colombiano se añadió un impuesto indirecto más a la larga lista de impuestos que conforman el sistema tributario colombiano.

De acuerdo con Valero-Varela (2007), el gravamen a los movimientos financieros es un impuesto indirecto de orden nacional ${ }^{9}$ que se aplica de forma inmediata a los usuarios de las entidades del sistema financiero colombiano que dispongan de recursos en las cuentas corrientes o de ahorros por alguno de los medios de retiro. La base gravable del impuesto se

${ }^{9} \mathrm{E}$ ámbito de aplicación es todo el territorio nacional y el titular de la deuda tributaria es la nación. 
determina por el valor total de la transacción que se vaya a realizar y, si es el caso, por los intereses asociados al valor principal; los encargados de retener los recaudos del gravamen son el Banco de la República, las entidades vigiladas por la Superintendencia Bancaria, las entidades vigiladas por la Superintendencia de Valores y las entidades vigiladas por la Superintendencia de Economía Solidaria. Estas entidades hacen las veces de agentes retenedores y deben declarar de manera semanal los montos recaudados a la Unidad Administrativa Especial Dirección de Impuestos y Aduanas Nacionales (DIAN).

Es importante precisar que si bien la formulación teórica de Tobin buscaba regular los movimientos de fondos, la medida que terminó por implementarse en Colombia dista de la formulación teórica en el objetivo que persigue, pues mientras la fórmula original propone prevenir las crisis por medio de una regulación de movimientos de los fondos, la propuesta de Colombia surge como respuesta a la crisis del sector bancario con el fin de solucionar los efectos de la falta de liquidez que pudiera tener el sistema financiero sobre la economía en ese momento. Por esta razón y dada la falta de un mercado estructurado y sólido de valores en Colombia el único modo de obtener contribuciones para los bancos era gravar las transacciones que estos registraban.

\section{El sistema tributario colombiano —impuestos indirectos y directos-}

La tributación ha sido un tema de interés para una larga serie de economistas que, incluso, se ha tratado desde David Ricardo en su obra Economía política y principios de tributación. Desde entonces todos concuerdan en estudiar las características y los principios que debe cumplir un sistema tributario óptimo. Uno de los economistas más reconocidos en este tema es Stiglitz (1997), quien inicialmente define los impuestos como transferencias obligatorias de los ciudadanos al Estado sin que exista una contraprestación específica; estas transferencias ocultan una relación de dominio que está amparada por la legalidad y el respeto que le confiere el proceso político. Adicionalmente, aclara que los impuestos tienen su origen en la creación de los Estados y que de ellos existen dos clases: los impuestos directos, los cuales se aplican sobre las personas naturales y las sociedades, y los impuestos Este artículo es un online first (versión definitiva del contenido del artículo, con diagramación provisional y asignación de Dol). Aún no cuenta con páginas definitivas, pero es citable utilizando su código DOI. 
indirectos, que se le aplican a una amplia cantidad de transacciones de bienes y servicios.

Sin embargo, esa estabilidad que profieren los impuestos al poder político no es neutral; Stiglitz (1997) señala que los impuestos tienen efectos directos sobre la economía de los países y sobre las decisiones económicas que toman los agentes en el mercado. El tipo de efectos pueden generar consecuencias positivas o negativas según sean las características y condiciones — estructura - del impuesto implementado. Por tanto, dentro de las políticas tributarias es muy importante tener en cuenta qué cambios puede sufrir el comportamiento económico de una sociedad como consecuencia de implementar nuevos gravámenes.

Ahora bien, si conocemos la importancia de evaluar la estructura de los impuestos, lo siguiente es definir las características del sistema. Stiglitz (1997) estableció algunas de las características básicas que debe cumplir cualquier sistema tributario. Entre estas se habla de la eficiencia económica, la cual sugiere que la tributación no puede interferir en la asignación eficiente de los recursos de la economía; la flexibilidad, es decir, debe adaptarse rápidamente a los cambios en el ciclo económico; la responsabilidad política, por la que se busca que el sistema sea transparente para los individuos y les permita así reconocer lo que están pagando y a quiénes benefician estos pagos; $y$, por último, la justicia, la cual indica que el trato a los individuos debe desprenderse de sus características o capacidad de pago.

Adicionalmente, explica las dos concepciones de equidad que existen dentro del sistema tributario de un país, las cuales deben ser claras a la hora de evaluar la estructura tributaria de una economía. En primer lugar, se tiene la definición de equidad horizontal: los individuos que son iguales en todos los aspectos relevantes deben tratarse igual; en segundo lugar, se habla de la equidad vertical: los individuos que se encuentran en mejores condiciones que otros - en términos de ingresos- deben tener una contribución impositiva más alta, es decir, un impuesto que cumple con el criterio de equidad vertical termina por ser progresivo, dada la definición de progresividad tributaria.

Alrededor de la conformación del sistema tributario y del tipo de impuestos que lo componen existe una serie de debates. El caso particular del GMF no es la excepción, ya que ha logrado trascender y ser motivo de controversias no solo en Colombia, sino en el mundo Este artículo es un online first (versión definitiva del contenido del artículo, con diagramación provisional y asignación de Dol). Aún no cuenta con páginas definitivas, pero es citable utilizando su código DOI. 
entero - a través de las diferentes variantes que se han adoptado-; es, por tanto, necesario repasar algunos de los trabajos que han contribuido a esta discusión. En la actualidad existen algunos estudios que analizan la concepción teórica de lo que debería ser el GMF, entre los cuales dos trabajos se destacan.

Primero, el de Romero-Londoño (2013), titulado Evolución del impuesto de gravamen a los movimientos financieros (GMF) desde su implementación a finales de la década de los noventa hasta la actualidad en Colombia; en este, aparte de presentar necesariamente los principios rectores de establecimiento del tributo, se da una connotación microeconómica al sostenimiento del impuesto a partir de hechos estilizados de corrupción que confluyen en un sostenimiento del tributo debido al fenómeno de rent seeking ${ }^{10}$, por encima de cualquier análisis práctico que pueda realizarse. Si bien el estudio toma un tinte político, permite tener presente un factor crucial en los tributos y es su carácter político.

Por otra parte, Matheson (2011), en Taxing financial transaction: issues and evidence, posibilita una óptica mucho más analítica con base en los diferentes tipos de gravamen sobre transacciones financieras que existen, los cuales se extienden a activos financieros específicos - bien sean bonos o fondos de inversión, entre otros-. Así, por medio de un analisis econométrico de elasticidades determina que si bien el impuesto implicará cambios sobre los precios de los activos mencionados, también ejercerá la labor de control sobre los mercados financieros, una afirmación que coincide con el propósito del tributo del GMF en Colombia de preservar la estabilidad del sistema financiero a nivel interno.

Por último, para mostrar las incidencias del GMF sobre la economía se han encontrado estudios acerca de su relación con la oferta monetaria, como, por ejemplo, el de Lozano y Ramos (2000), «Análisis sobre la incidencia del impuesto del 2 x 1000 a las transacciones financieras». Estos autores encuentran que después de afirmar el carácter progresivo del impuesto, debido a la composición teórica bajo la cual se implementó, establecen un análisis econométrico para estimar un grado de correlación entre el incremento en la demanda por

\footnotetext{
${ }^{10}$ Búsqueda de rentas o ingresos para su propio beneficio originados de transferencias estatales por parte de los representantes políticos.

Este artículo es un online first (versión definitiva del contenido del artículo, con diagramación provisional y asignación de DOI). Aún no cuenta con páginas definitivas, pero es citable utilizando su código DOI.
} 
efectivo del público y el establecimiento del tributo. Así explican un efecto de causalidad de la entrada en vigencia del gravamen con el incremento en la demanda por efectivo.

De igual forma, Pacheco (2017), en «El gravamen a los movimientos financieros: análisis del recaudo tributario versus la oferta monetaria en Colombia», empleando una metodología menos sofisticada por medio de análisis de tendencias de los principales agregados monetarios y el comportamiento del tributo a lo largo de su entrada en vigencia, corrobora lo concluido por Lozano y Ramos (2000), de tal suerte que el GMF presenta afectaciones de la oferta monetaria, expresada a través de las variables M1 y M2. También Pacheco (2017) afirma que el establecimiento del tributo es un incentivo bastante fuerte para evitar el acceso al sistema financiero basado en la preferencia por liquidez y el costo adicional que implica el tributo.

Por otra parte, es importante caracterizar el indicador de progresividad/regresividad, por lo que se revisó el trabajo de López et al. (2011), titulado «La Constitución de 1991 y sus implicaciones en materia tributaria y de equidad. Una aproximación a la medición de la progresividad en Colombia». En este documento los autores realizan un análisis sobre los impuestos indirectos y su carácter regresivo, de manera que aportan hechos y pruebas verificables sobre el comportamiento que deben seguir a nivel teórico los impuestos indirectos. En este sentido, es una fuente de contraste y ayuda teórica sobre el carácter regresivo que, por definición, debe cumplir el GMF, ya que es catalogado como un impuesto indirecto.

\section{Metodología}

Se utilizan como guía para la formulación y la aplicación de los indicadores dos trabajos, principalmente. El primero de ellos, sobre la productividad del GMF, realizado por Valero-Varela (2007). Por otro lado, a fin de encontrar la caracterización del indicador de progresividad/regresividad, es necesario remontarse al trabajo de López et al. (2011). Para el caso de los datos de recaudo por tipo de impuesto y recaudo total que ha tenido el Gobierno nacional a través de la DIAN se toman los datos del periodo de análisis 1999-2018, debido a que la serie considera datos continuos y, bajo la misma metodología, para el periodo 1970Este artículo es un online first (versión definitiva del contenido del artículo, con diagramación provisional y asignación de DOI). Aún no cuenta con páginas definitivas, pero es citable utilizando su código DOI. 
2020. En el caso del coeficiente de Gini se emplean, en conjunto los datos que ofrece el Banco Mundial en su anexo de indicadores para Colombia y con los anexos de pobreza que entrega el DANE. Por último, es necesario recurrir a la serie de datos del DANE del IPC o Índice de Precios al Consumidor ${ }^{11}$ año base 2005 para unificar las series y así construir los datos del periodo 1999-2018.

De igual forma, en el caso del PIB nominal para Colombia, debido a los cambios de metodología, es necesario realizar un empalme con el propósito de consolidar los datos a una misma base metodológica que, en el caso puntual, será 2005. De acuerdo con la Dirección de Síntesis y Cuentas Nacionales (2013), el método correcto es el de interpolación por tasa de variación, pues permite conservar las características de la serie a través del tiempo, por lo que es necesario introducir la ecuación 3.1 que describe el proceso a realizar con miras a establecer los datos a una misma base.

$$
V n_{B}^{t=B-1}=\frac{V n_{B}^{t=B} \times V n_{b}^{t=B-1}}{V n_{b}^{t=B}}
$$

Donde:

- $V n$ representa el valor nominal;

- $B$ es la base nueva, o año base con el que se desea trabajar;

- $b$ es la base anterior o la base en la que se desea hacer el empalme;

- $t$ es el año en consideración.

Por otra parte, si se tiene en cuenta que la serie de datos del índice Gini tiene algunos datos faltantes, se debe realizar una imputación de valores para los años 2006 y 2007, los cuales se omitieron debido al cambio metodológico que realizó el DANE. En este proceso se realiza un gráfico de los valores que se tienen para el Gini, empleando el software Excel, se traza la tendencia con promedios de media móvil de dos periodos que permiten un mejor

\footnotetext{
11 Indicador del nivel de precios de una canasta de bienes y servicios para un periodo de tiempo específico. Este artículo es un online first (versión definitiva del contenido del artículo, con diagramación provisional y asignación de DOI). Aún no cuenta con páginas definitivas, pero es citable utilizando su código DOI.
} 
ajuste a la distribución de datos y, por esta vía, se realiza una estimación detallada de los valores faltantes, evitando incurrir en sub o sobreestimaciones que puedan afectar el análisis y desarrollo del trabajo.

\subsection{Cálculo del indicador de productividad}

El indicador de productividad de un impuesto, de acuerdo con Valero-Varela (2007), no es más que la relación entre el recaudo en términos del producto interno bruto con respecto a la tasa efectiva. Para el cálculo del indicador se sigue la metodología empleada por Steiner y Soto (1998), mediante la siguiente ecuación:

$$
\rho=\frac{R}{\tau}(1.1)
$$

De la anterior ecuación es necesario precisar que:

- $\rho$ hace referencia a la tasa de productividad del impuesto;

- $R$ es el recaudo del gravamen en términos del PIB (producto interno bruto);

- $\tau$ es la tasa efectiva cobrada en términos porcentuales.

Al tener en cuenta la fórmula para el cálculo del indicador de productividad del GMF es necesario extraer de la serie de datos construidos las variables de recaudo del GMF, el PIB nominal y, por último, la tarifa efectiva que a partir del 2003 ha sido constante. Una vez extraídas las variables se aplica la ecuación 1.1. Así mismo, se hace necesario realizar una precisión sobre esta ecuación, por lo cual se introduce la ecuación 1.2 para aclarar el cálculo de R, tal como se muestra a continuación:

$$
R=\frac{r}{Y}(1.2)
$$

Donde $R$ se establece como el recaudo del tributo en términos del PIB, $r$ es el valor nominal del recaudo del tributo para un año en particular, e $Y$ es el PIB en términos 
nominales para un año en particular. Valores más altos de $R$ sugieren mayor productividad que pueden verse afectados bien sea por aumentos en la tasa de tributación o bien por fluctuaciones negativas del recaudo como porcentaje del PIB.

\subsection{Cálculo del indicador de progresividad/regresividad}

De acuerdo con López et al. (2011), la forma más sencilla de analizar el impacto de progresividad es a través del método de progresión por participación, el cual permite analizar el carácter de un impuesto en particular. El indicador se expresa de la siguiente manera:

$$
M P P_{i, t}=\frac{\partial G_{t}\left(Y_{t}, R_{t}, \ldots\right)}{\partial\left(T_{i, t} / R_{t}\right)}
$$

Donde:

- $M P P_{i, t}$ hace referencia al indicador de metodología de progresión por participación —que determina el carácter progresivo o regresivo-;

- $G_{t}$ hace referencia al coeficiente de $G^{2} i^{12}$ en el año $t$ como una medida de desigualdad;

- $T_{i, t}$ es el recaudo obtenido en el año $t$ para un impuesto $i$ en particular;

- $R_{t}$ representa el recaudo total en el periodo $t$ por concepto de impuestos.

A fin de realizar el cálculo del indicador se requiere extraer de la serie de datos construida las variables del coeficiente de Gini el recaudo del GMF y el recaudo total por concepto de impuestos a nivel nacional. Una vez extraídas las variables siguiendo la ecuación 2.1 se requiere el cálculo de la participación relativa del GMF con respecto a los impuestos, siendo este el cociente entre el recaudo del GMF con respecto al recaudo total. Al introducir las ecuaciones 2.2 y 2.3 se obtienen los criterios de clasificación del indicador que se explican más adelante.

\footnotetext{
12 Coeficiente de desigualdad que mide la relación entre la curva de Lorenz y la curva de equidistribución: cercano a 1 mayor desigualdad, cercano a 0 mayor equidad.

Este artículo es un online first (versión definitiva del contenido del artículo, con diagramación provisional y asignación de DOI). Aún no cuenta con páginas definitivas, pero es citable utilizando su código DOI.
} 


$$
\begin{aligned}
& M P P_{i, t}=\frac{\partial G_{t}\left(Y_{t}, R_{t}, \ldots\right)}{\partial\left(T_{i, t} / R_{t}\right)}<0(2.2) \\
& M P P_{i, t}=\frac{\partial G_{t}\left(Y_{t}, R_{t}, \ldots\right)}{\partial\left(T_{i, t} / R_{t}\right)}>0(2.3)
\end{aligned}
$$

De esta forma, el indicador mide variaciones de la relación entre desigualdad y tributación, a fin de marcar la tendencia de los resultados y afirmar patrones de progresividad o regresividad. Por esta razón, se consideran fundamentalmente dos posibles escenarios. En el primero de ellos las variaciones ya sea en el Gini o en el recaudo relativo del impuesto son negativas, no de manera simultánea, es decir, ambas fluctuaciones deben presentar signos de efectos contrarios; allí se considera que hay progresividad, es decir, que la desigualdad ha presentado una disminución con base en la evolución del tributo.

El segundo escenario sugiere que las variaciones del Gini o el recaudo con un signo positivo, y como condición necesaria, requiere que el signo del efecto sea igual de manera simultánea, es entonces que se puede afirmar que hay regresividad por presentar bajos cambios sobre la desigualdad al cambiar el recaudo. Este indicador se basa en análisis de tendencia, pues emplea los cambios que se presentan a través de los años. Así mismo, es una aproximación que al emplear derivadas supone de entrada que el resto de variables permanecen constantes; sin embargo, a nivel del Gini no tiene en cuenta cualquier tipo de factor externo que la afecta, bien sea el crecimiento económico o bien políticas públicas de lucha contra la pobreza, entre otras.

Finalmente, para analizar el comportamiento de los indicadores mencionados es necesaria la construcción de gráficos que muestren la forma en la que se han comportado los indicadores. Así mismo, es necesario introducir la línea de tendencia en los gráficos que permitan consolidar el comportamiento que han seguido los indicadores. Adicionalmente, con respecto al indicador de regresividad es necesario incluir el gráfico de nube de puntos que contrasta el Gini en el eje vertical y la participación relativa del GMF en el recaudo total, de la cual se espera que la línea de tendencia se comporte de la misma manera que la del indicador de regresividad. 


\section{Resultados}

\subsection{Productividad del GMF}

\subsubsection{Valoración con cifras nominales.}

La figura 1 presenta el comportamiento del indicador de productividad del GMF entre 1999 y el 2018. El resultado obtenido es consistente con los resultados que obtuvo ValeroVarela (2007), afirmando una disminución en la productividad hasta el 2007. Esto, dado que el recaudo por concepto de este tributo dependerá del costo de usar el sistema financiero, pues entre mayor sea la tasa del GMF implicará una mayor probabilidad de no usar las transacciones financieras y, por tanto, se dejará de usar el sistema, llevando la tendencia de recaudo a puntos más bajos. Esta situación se extendió hasta el 2010. Sin embargo, a partir de ese mismo año se presentó una tendencia creciente y estable en el orden de 0,2, lo que contrastaba con el 0,25 inicial, el cual no aumentó después del 2010.

Figura 1. Comportamiento del indicador de productividad del GMF entre 1999 y el 2018

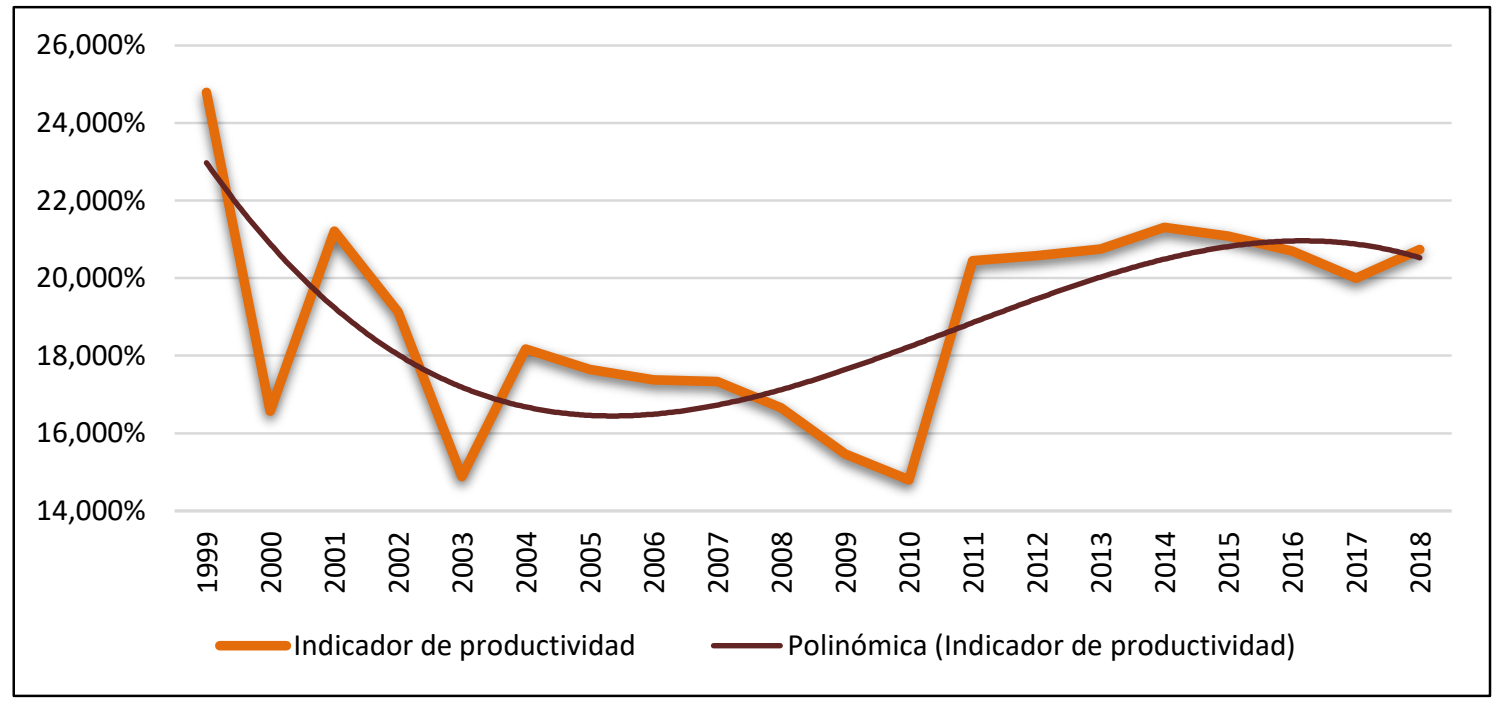

Fuente. Elaboración propia con base en datos del DANE y la DIAN. 
Por su parte, el análisis de tendencia sugiere un aumento en la productividad del gravamen que coincide con lo mostrado en la figura 2, el cual expresa el GMF como porcentaje del PIB. La tendencia ha venido en aumento, acercándose al 1 \% del PIB, situación que implica un buen ejercicio de recaudo si se considera que no se ha presentado evolución de la tarifa a partir del 2003, la cual es del 0,4\%.

Figura 2. Comportamiento del recaudo del GMF como porcentaje del PIB nominal

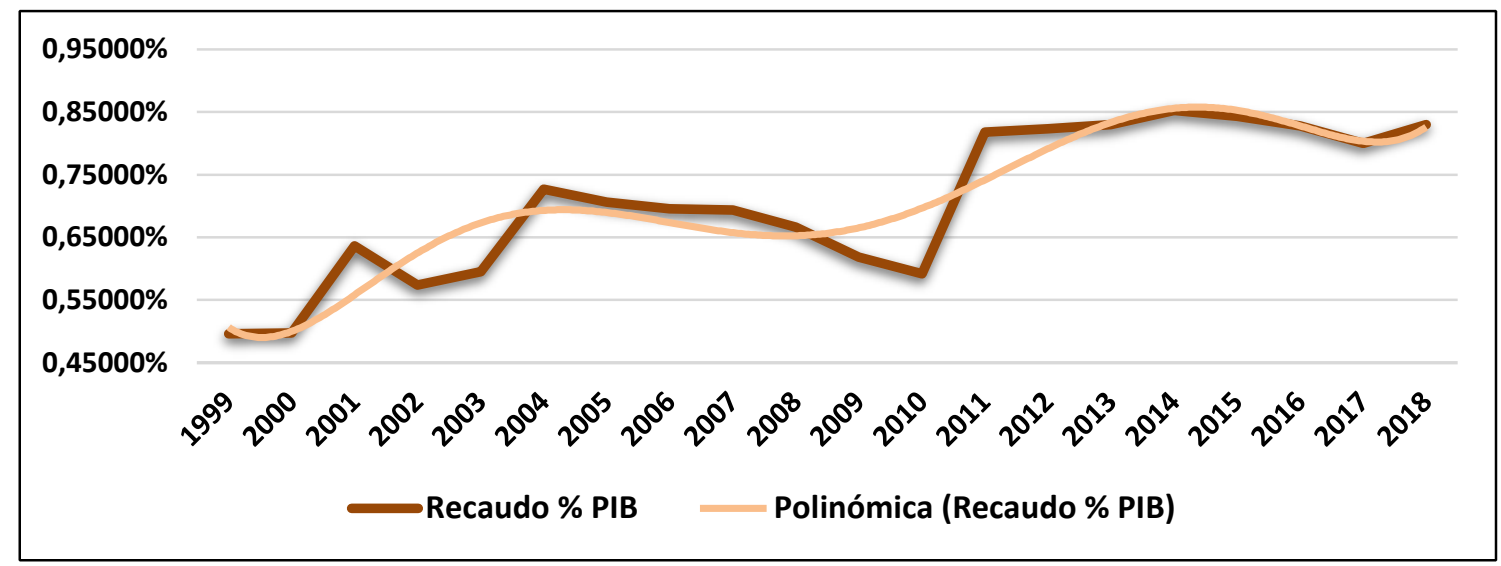

Fuente. Elaboración propia con base en datos del DANE y la DIAN.

Para entender el ascenso en el recaudo recurrimos al análisis de Cavallo y Powell (2019), quienes indican que las decisiones de política monetaria de los países con economías más desarrolladas tienen repercusiones sobre los flujos de capitales en los mercados emergentes y permiten así el desarrollo de los sistemas financieros. Por ejemplo, sugieren que después de la crisis del 2008 aumentó la liquidez internacional, lo que les permite a los mercados emergentes fortalecerse y asumir nuevos capitales a partir del diferencial en tasas de interés.

Esta tesis es respaldada por el Banco de la República (2019), entidad que sugiere que la tendencia creciente en la participación del sector financiero es una prueba de esto, pues se refleja en el incremento de las utilidades de los bancos, las cuales, según Portafolio (2019), fueron de COP 1 793,3 billones de pesos en abril del 2019, es decir, COP 165,6 billones de pesos más en contraste con el 2018. Todo esto respalda la idea de que la bancarización o el 
fortalecimiento del sistema financiero, bien sea por movimientos de política externa o bien producto de la mayor demanda de productos financieros para el manejo de recursos han conducido a un mayor recaudo del tributo.

\subsection{Valoración con cifras reales o a precios constantes de 2005}

Figura 3. Comportamiento del indicador de productividad 1999-2018

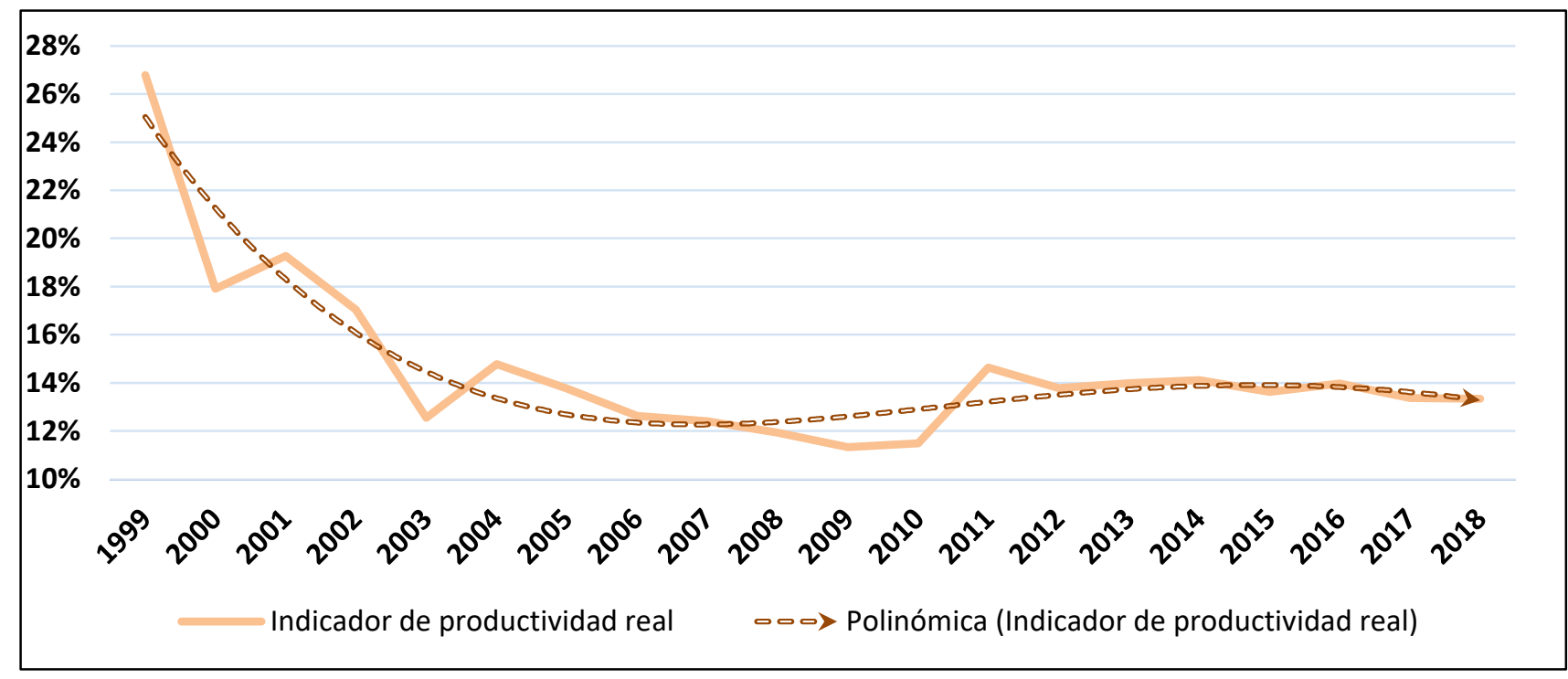

Fuente. Elaboración propia con cálculos propios y cifras del DANE.

Al hacer el ejercicio con cifras reales, es decir, descontando el efecto inflacionario sobre las variables, se obtiene un comportamiento diferente al mostrado más arriba. Con esta nueva tendencia se puede evidenciar una caída significativa y constante de la productividad del GMF para el periodo en cuestión. Esa caída constante puede explicarse por un decaimiento en el recaudo del tributo ocasionado por las múltiples exenciones al pago del impuesto y los efectos negativos de su sostenimiento, el cual genera incentivos a actividades informales, lo que incrementa la evasión y elusión del impuesto.

Otro factor a considerar es el efecto Olivera-Tanzi, pues el efecto inflacionario juega un papel muy importante en el análisis de las cifras, ya que afecta negativamente el valor real del recaudo y lleva a que el gobierno reciba un monto real menor de recaudo respecto a las 
erogaciones pasadas. Esto queda en evidencia con el comportamiento de los gráficos antes y después de eliminar la inflación (Svaljek y Anušić, 1996).

El cambio, la tendencia del indicador de productividad también pude mostrar que el aumento concluido anteriormente no se debe necesariamente al incremento en los niveles de recaudo, pues como se puede ver en la figura 4 el nivel de recaudo del GMF como porcentaje del PIB real no ha tenido un crecimiento importante durante el periodo de análisis, e incluso permite concluir estabilidad en el recaudo del GMF. El comportamiento del recaudo, entonces, nos lleva a ultimar que el aparente aumento de la productividad del GMF es más bien efecto inflacionario que buenas prácticas de recaudo.

Figura 4. Comportamiento del recaudo del GMF como porcentaje del PIB real

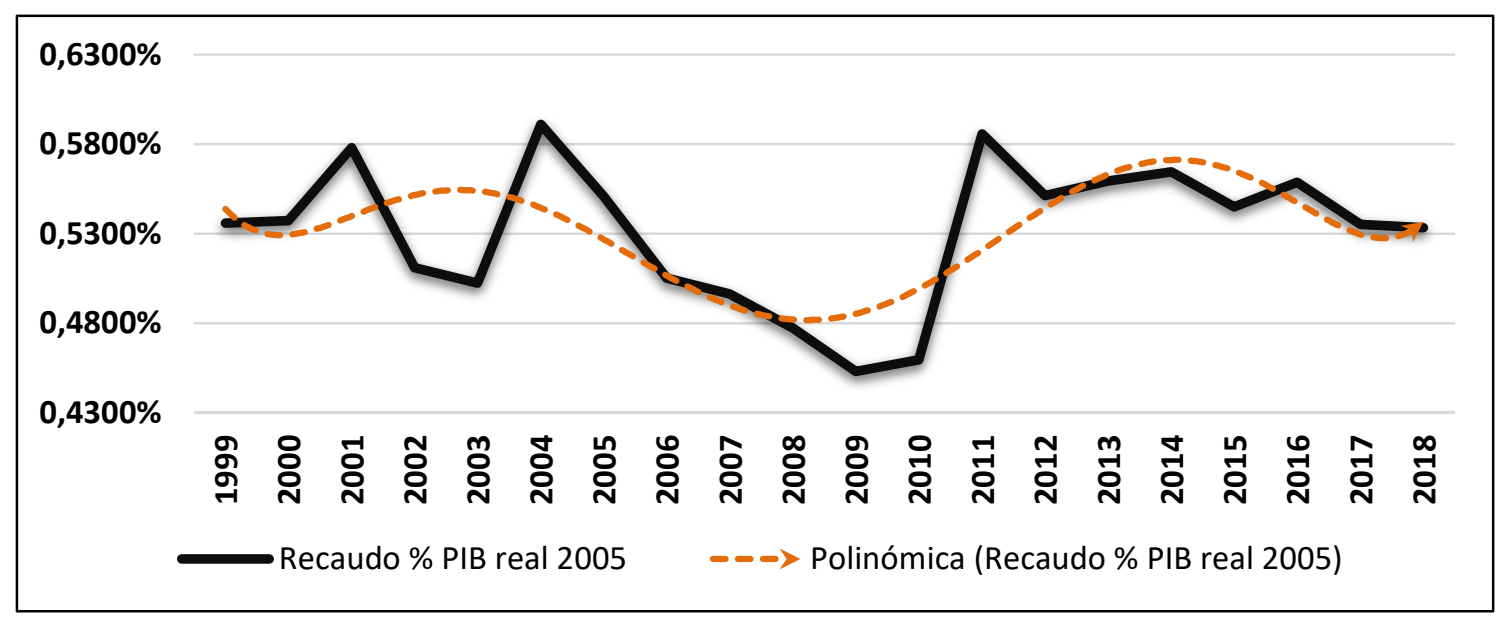

Fuente. Elaboración propia con cálculos propios y cifras del DANE. 


\subsection{Regresividad del GMF}

\subsubsection{Valoración con cifras nominales.}

La figura 5 revela el comportamiento del indicador de progresividad del GMF entre 1999 y el 2018, caracterizándolo como un impuesto progresivo, dada la tendencia negativa que exhibe. Sin embargo, se han presentado algunas fluctuaciones importantes. La primera de ellas durante el periodo 2004-2009, que evidencia un incremento sostenido en términos de regresividad, situación que puede relacionarse, principalmente, con el aumento de la tasa del 3 x 1000 al 4 x 1000 en el 2004 y la instauración del gravamen como permanente en el 2006. La segunda fluctuación importante se da en el 2010, la cual marca la caída en la tendencia indicando progresividad y, a partir de allí, un aumento constante en esta. Ese aumento sostenido se debe, en cierta medida, a la modernización del sistema tributario frente a las prácticas de elusión y evasión.

Figura 5. Comportamiento del indicador de progresividad del GMF entre 1999 y el 2018

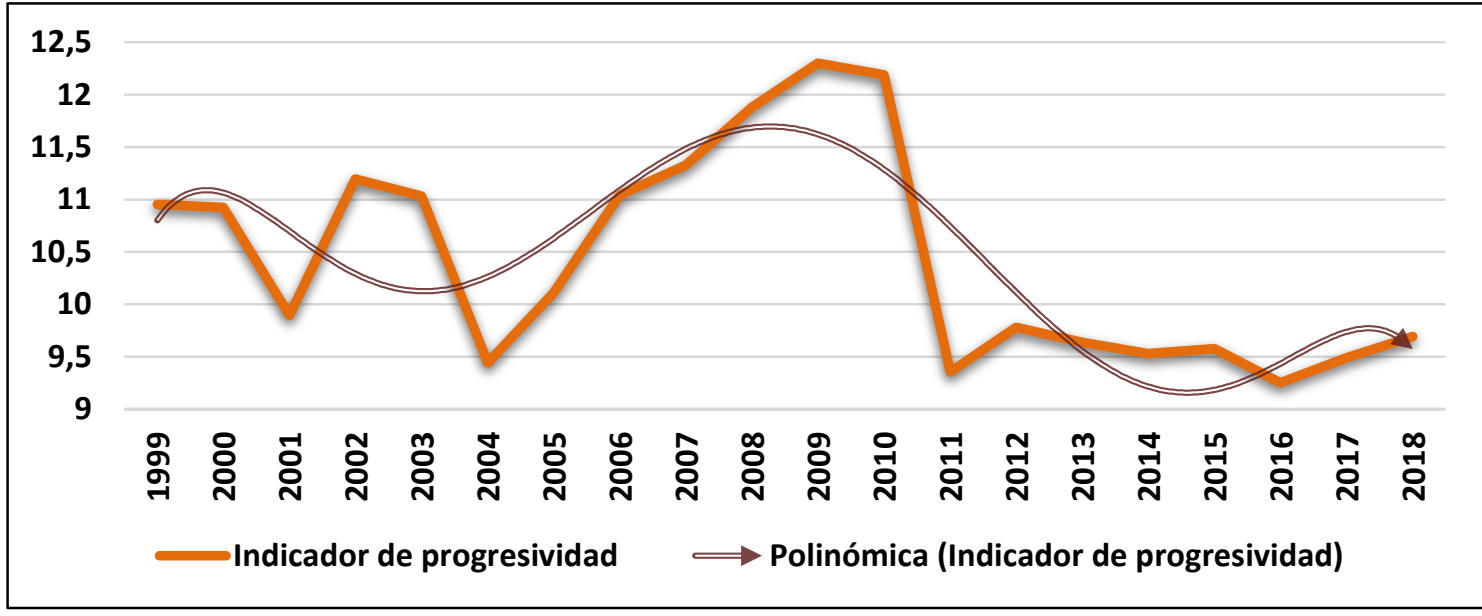

Fuente. Elaboración propia con base en datos del DANE y la DIAN.

Otro de los factores que explica los resultados obtenidos proviene de un estudio del DANE (2006), señalando que los hechos generadores del GMF se concentran en la población bancarizada. Por lo general, de los estratos 4, 5 y 6, que son los de mayor capacidad de pago y acceden más fácilmente al sistema financiero. Así mismo, las excepciones a hechos generadores, por ejemplo, la tenencia de una cuenta de ahorros y la exención de pago por 
montos mensuales menores a 350 UVT $^{13}$ genera beneficios a personas de estratos 1,2 y 3 , lo que contribuye al objetivo de progresividad. La contribución del GMF con la equidad y la reducción de la desigualdad puede evidenciarse en el comportamiento del GINI para Colombia después de la implementación del gravamen.

La figura 6 muestra la evolución del Gini, dado el comportamiento de recaudo del GMF para el periodo 1999-2018. Este sugiere una tendencia negativa e indica un comportamiento progresivo, es decir, al aumentar la participación del GMF en el recaudo tributario total, el índice Gini disminuye. Estos resultados comprueban que los impuestos indirectos no necesariamente tienen comportamiento regresivo y que, en el caso puntual del GMF, su participación contribuye en una pequeña proporción con la reducción de la desigualdad en el país.

Figura 6. Evolución del GINI respecto al recaudo en cifras nominales del GMF en Colombia -19992018-

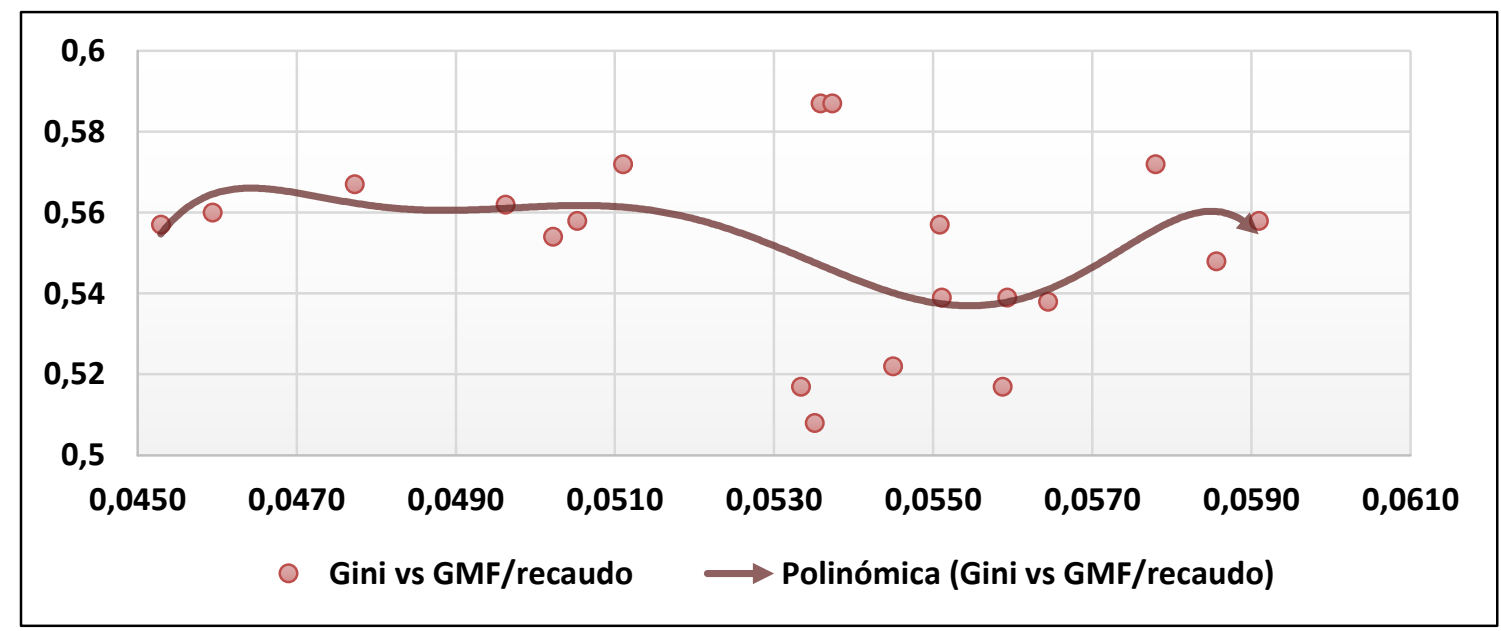

Fuente. Elaboración propia con base en datos del DANE y la DIAN.

\footnotetext{
13 Unidades de valor tributario.

Este artículo es un online first (versión definitiva del contenido del artículo, con diagramación provisional y asignación de DOI). Aún no cuenta con páginas definitivas, pero es citable utilizando su código DOI.
} 


\subsubsection{Valoración con cifras reales o a precios constantes del 2005.}

Respecto a la progresividad, al trabajar cifras deflactadas la tendencia se mantiene, es decir, después de restar el efecto inflación, el GMF exhibe aún un comportamiento progresivo. Según Svaljek y Anušić (1996), se debe a que un impuesto progresivo, por lo general, no se ve afectado en su estructura por los efectos inflacionarios. El comportamiento progresivo es más evidente desde el 2010 debido al intenso proceso de bancarización llevado a cabo en la última década en Colombia, en reacción a la crisis del 2008 y al alto flujo de capitales hacia economías emergentes.

La figura 7 permite confirmar la progresividad del GMF en términos reales y afirmar que este impuesto indirecto contribuye a la reducción de la concentración del ingreso en la economía colombiana, incluso después de eliminar el efecto por inflación. De acuerdo con Narob (2015), existe una relación positiva pero no lineal con la distribución del ingreso, es decir, a mayor inflación en las economías en desarrollo se presenta mayor desigualdad en la distribución del ingreso.

Figura 7. Comportamiento del indicador de progresividad del GMF con cifras a precios constantes del 2005 entre 1999 y el 2018

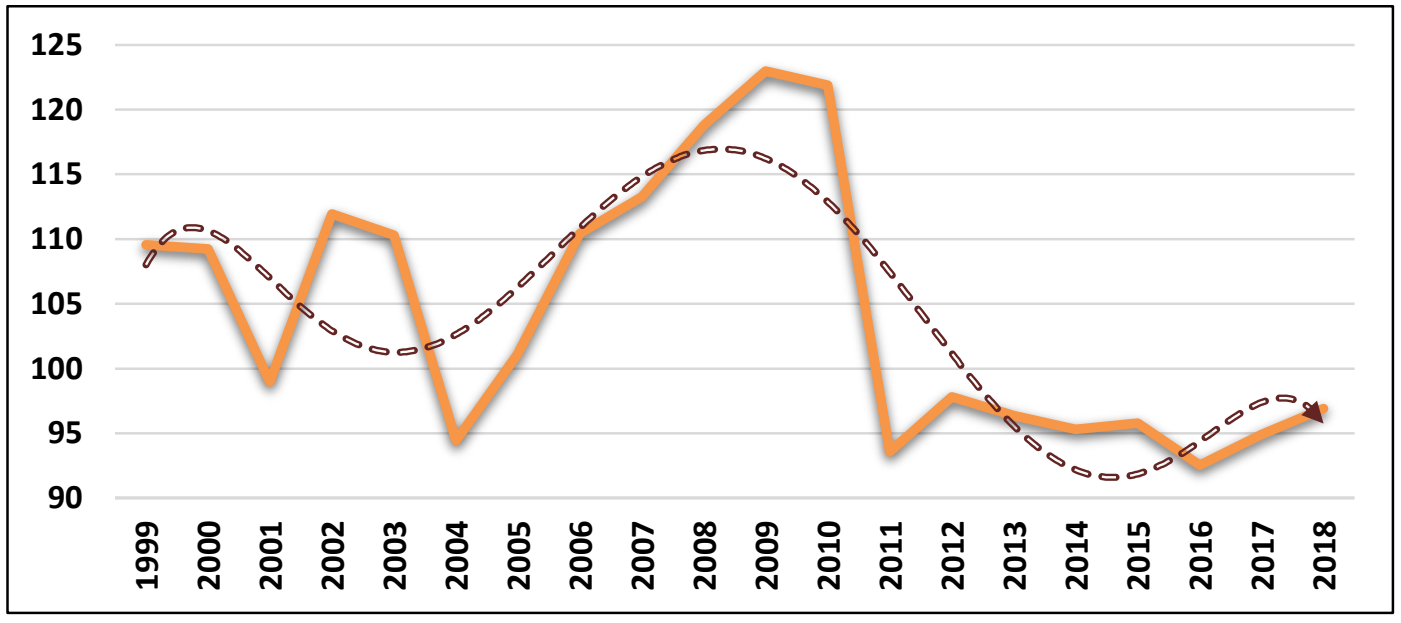

Fuente. Elaboración propia con cálculos propios y cifras del DANE. 
Así que, dadas las condiciones de la economía colombiana, esta validación permite esclarecer que instrumentos como este impuesto permiten cumplir con el principio constitucional de progresividad impositiva al mostrar que el GMF, efectivamente, es un impuesto que, a pesar de ser indirecto y tener una tarifa plana sin excepciones, cumple el principio de progresividad tributaria y contribuye a la reducción de las brechas en la economía colombiana.

Figura 8. Evolución del Gini respecto al recaudo en cifras a precios constantes de 2005 del GMF en Colombia -1999-2018-

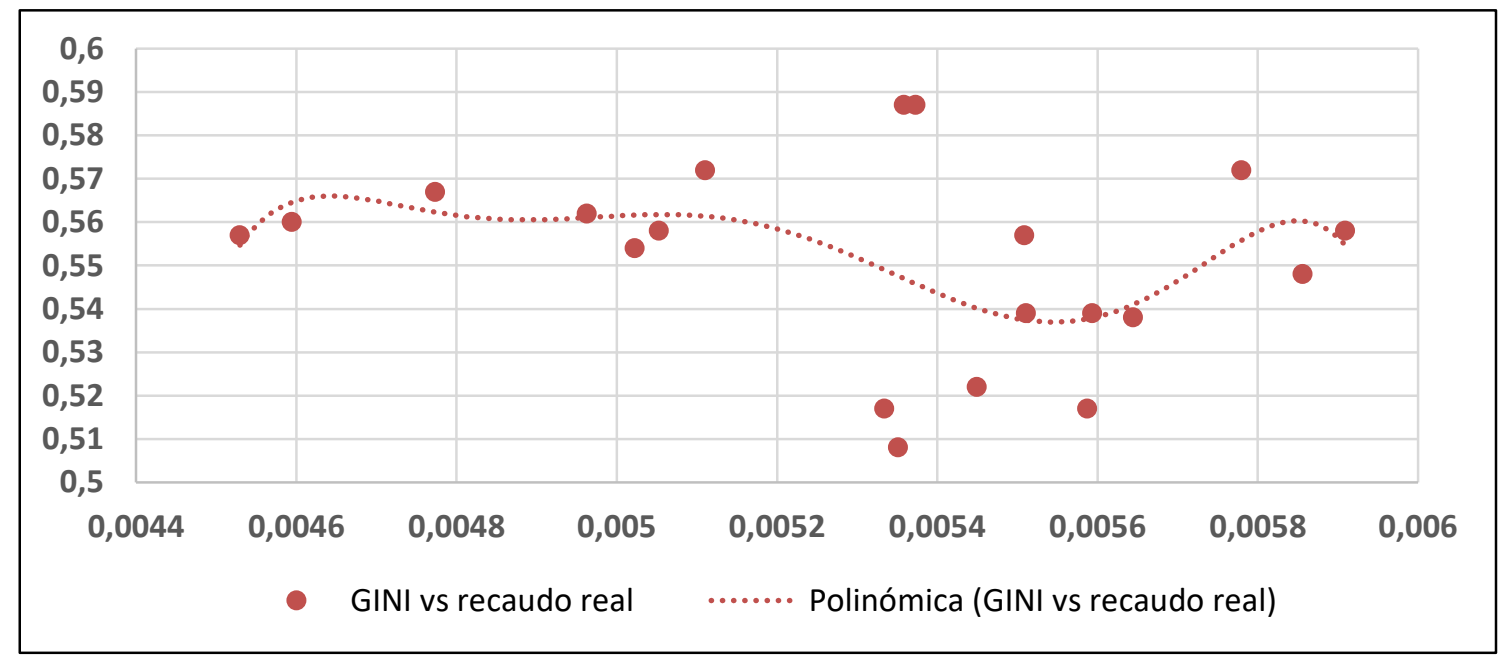

Fuente. Elaboración propia con cálculos propios y cifras del DANE.

\section{Conclusiones}

La importancia del sistema financiero en Colombia, en general, viene en ascenso. Es este comportamiento el que ha generado un complejo entorno de discusión alrededor del sostenimiento del GMF, una discusión que, hasta el momento, ha sido enmarcada en un entorno analítico que recurre de manera permanente a facultades teóricas y se distancia de los aspectos empíricos. Por esta razón, se sugiere contrastar la suposición teórica con indicadores sujetos a datos reales que conduzcan a la identificación del comportamiento real del tributo y permitan visualizar el objetivo que justifica la transición que tuvo el impuesto durante su implementación de transitorio a permanente. Es por esta razón que se hace 
necesario estudiar si el GMF cumple con los principios tributarios que establece la Constitución Política a través del cálculo de los indicadores de productividad y de progresividad para el periodo 1999-2018.

El resultado obtenido para el caso del indicador de productividad, en términos nominales, es consistente con los resultados obtenidos por Valero-Varela (2007), quien afirma una disminución en la productividad hasta el 2007 gracias al aumento de la tasa efectiva de recaudo del impuesto. Sin embargo, si bien el análisis de tendencia sugiere un aumento en la productividad del gravamen, este hecho es ampliamente contrastado, ya que la propuesta teórica del tributo supone facilidad en el recaudo al evidenciarse esta en el buen ejercicio de recaudo en términos del PIB.

Así mismo, el incremento de la participación del GMF en el PIB se puede atribuir no solo a los flujos de fondos a nivel internacional, sino que también se debe tener en cuenta el componente interno del sistema financiero, el cual juega un papel importante, ya que se está dando un proceso de bancarización en el que las personas recurren cada vez más al sector financiero en la cotidianidad, lo que lleva a que por esta vía el recaudo del GMF aumente. Esta bancarización, tal como lo expone la Superfinanciera (2019), ha permitido que el indicador de inclusión financiera se situé en 81,4 \% para el 2018.

Esto implica que ese porcentaje de la población colombiana tiene, por lo menos, un producto financiero, situación que contrasta con los resultados de Lozano y Ramos (2000) y Pacheco (2017), es decir, esos procesos de bancarización pueden estar influenciados por una mayor demanda de efectivo por parte del público, lo cual, según los autores, implica correlación entre el aumento de la demanda por efectivo y la entrada en vigencia del tributo. En conclusión, se podría indicar que las fluctuaciones del recaudo de este tributo podrían estar más ligadas al hecho generador de este - transacciones financieras- que a temas administrativos del recaudo.

Por su parte, el resultado del indicador de progresividad, en términos nominales, sugiere que, en general, el GMF se ha caracterizado por ser un impuesto progresivo. Este aumento sostenido en la progresividad del gravamen se debe, por una parte, a los hechos Este artículo es un online first (versión definitiva del contenido del artículo, con diagramación provisional y asignación de DOI). Aún no cuenta con páginas definitivas, pero es citable utilizando su código DOI. 
generadores del impuesto, los cuales los realizan, principalmente, personas con alta capacidad económica, así como a los progresos en la eficiencia del sistema administrativo, a la reducción en actividades de evasión y elusión del impuesto y a la larga lista de exenciones que están contempladas en el artículo 879 del estatuto tributario, como, por ejemplo, las operaciones de liquidez del Banco de la República con el Gobierno, la exención para cuentas con movimientos menores a las 350 UVT — cerca de once millones de pesos colombianos-, los créditos con destinación educativa emitidos por el Icetex - Instituto Colombiano de Crédito Educativo y Estudios Técnicos en el Exterior-, las operaciones de la Dirección de Tesoro Nacional a los órganos ejecutores, entre otros (Congreso de la República, 1989).

La contribución del GMF a la equidad y a la reducción de la desigualdad se puede evidenciar en el comportamiento que ha tenido el índice de Gini para Colombia después de la implementación del gravamen para el periodo 1999-2018. Sin embargo, el ejercicio de contrastar los indicadores obtenidos a partir de variables nominales con indicadores en términos de variables reales con el objetivo de eliminar el efecto inflacionario para establecer de esta manera un análisis más robusto y preciso del comportamiento real del GMF, así como eliminar el efecto de la inflación sobre la conducta de las finanzas públicas, permitió confirmar el resultado obtenido en el indicador de progresividad.

Dicho indicador sugiere que el GMF, efectivamente, es un impuesto progresivo que contribuye a la reducción de la concentración del ingreso a pesar de ser indirecto, de no tener en cuenta la capacidad de pago de los contribuyentes y de tener una tarifa plana sin excepciones. El GMF es un impuesto que cumple el principio de progresividad tributaria y contribuye a la reducción de las brechas en la economía colombiana.

Por su parte, el indicador de productividad arrojó un comportamiento diferente después de deflactar las variables y eliminar el efecto inflacionario, pues evidencia una reducción de la productividad constante durante todo el periodo analizado y no solo hasta el 2007. Esta baja eficiencia en el recaudo se explica, en cierta parte, por el efecto Olivera-Tanzi, el cual manifiesta cómo la inflación afecta negativamente el valor real del recaudo y lleva a que el Gobierno reciba un monto real menor de recaudo respecto a las erogaciones pasadas. 
Esto queda en evidencia con el comportamiento de los gráficos antes y después de eliminar la inflación, los cuales confirman que el GMF no cumple con el principio constitucional de eficiencia.

En conclusión, el GMF es un impuesto que a lo largo de su evolución se ha acomodado a los resultados redistributivos del mercado y se caracteriza por tener una elasticidad ingreso-precio mayor a uno, es decir que es progresivo porque conduce a que la variación del precio sea menor a la variación del ingreso de los contribuyentes, retirando las dudas de la larga serie de trabajos teóricos citados a lo largo del documento, de manera que la evidencia empírica da razón a la existencia y el sostenimiento del tributo, que en concordancia con el estatuto tributario colombiano cumple los principios de progresividad y equidad, pero no con el principio constitucional de eficiencia, pues su recaudo ha permanecido sin aumentos significativos desde su implementación.

\section{Referencias}

Banco de la República. (2019). Informe de la junta directiva al congreso de la República. Recuperado de https://bit.ly/3qG58ew

Cavallo, E.; Powell, A. (2019). La normalización monetaria: impactos en los mercados de capitales y en los flujos de capital. En Construir oportunidades para crecer en un mundo desafiante. (1125). Washington: BID.

Congreso de la República. (1989). Estatuto tributario. Bogotá: Senado de la República. Recuperado de https://bit.ly/3AiEgWF

Cornford, A. (1996). The Tobin tax: Silver bullet for financial volatility, global cash cow or both? Nueva York: Naciones Unidas.

Departamento Administrativo Nacional de Estadísticas - DANE (2006). Estadísticas de NBI. Recuperado de https://bit.ly/3dAXKfc

Dirección de Síntesis y Cuentas Nacionales. (2013). Documento metodológico y resultados de la retropolación 1975-2005 base 2005. Bogotá: DANE. Recuperado de https://bit.ly/3wfDfLs

López, M.; Torres, E. E.; Molina, C. A. (2011). La Constitución de 1991 y sus implicaciones en materia tributaria y de equidad. Una aproximación a la medición de la progresividad en Colombia. Perfil de Coyuntura Económica, (17), 51-71. Recuperado de https://bit.ly/3hxqUNo

Lozano, I.; Ramos, J. (2000). Análisis sobre la incidencia del impuesto del 2 × 1000 a las transacciones financieras. Bogotá: Banco de la República. Recuperado de https://bit.ly/3xgOLaR 
Matheson, T. (01 de marzo de 2011). Taxing financial transactions: Issues and evidence (working paper 11/54). FMI. DOI: https://doi.org/10.5089/9781455220984.001

Narob, N. (2015). Income Inequality and inflation in developing countries: an empirical investigation. Economics Bulletin, 35(4), 2888-2902. Recuperado de https://bit.ly/36bcSM8

Pacheco, G. A. (2017). El gravamen a los movimientos financieros: Análisis del recaudo tributario versus la oferta monetaria en Colombia. Ad-Gnosis, 6(6), 177-193. DOI: https://doi.org/10.21803/adgnosis.v6i6.198

Portafolio. (10 de junio de 2019). Utilidades del sistema financiero aumentaron en abril. Recuperado de https://bit.ly/3Ay28pk

Romero-Londoño, L. F. (2013). Evolución del impuesto de gravamen a los movimientos financieros (Tesis de especialización). Universidad Militar Nueva Granada. Bogotá, Colombia. Recuperado de https://bit.ly/2TyBY4S

Steiner, R.; Cañas, A. (2013). Tributación y equidad en Colombia. Bogotá: Fedesarrollo.

Steiner, R.; Soto, C. (1998). IVA: Productividad, evasión y progresividad. Bogotá: Fedesarrollo. DOI: https://doi.org/10.2139/ssrn.83968

Stiglitz, J. (1997). La economía del sector público. Barcelona: Antoni Bosch Editor.

Superintendencia financiera. (11 de junio de 2019). Indicador de inclusión financiera subió al 81,4\%: ya son 28 millones de adultos con por lo menos un producto financiero. Recuperado de https://bit.ly/3dDnJTb

Svaljek, S.; Anušić, Z. (1996). Olivera-Tanzi effect: theory and its manifestation in the Croatian stabilization programme. Croatian Economic Survey, (3), 73-102. Recuperado de https://bit.ly/3dznbxB

Valero-Varela, H. J. (2007). Generalidades del gravamen a los movimientos financieros (GMF) en Colombia. Bogotá: DIAN. Recuperado de https://bit.ly/3jKkxJg 